\title{
Nonstationary Wavelets Related to the Walsh Functions
}

\author{
Yuri A. Farkov, Evgeny A. Rodionov \\ Department of Mathematics, Russian State Geological Prospecting University, Moscow, Russia \\ Email: farkov@list.ru
}

Received March 29, 2012; revised April 25, 2012; accepted May 2, 2012

\begin{abstract}
Using the Walsh-Fourier transform, we give a construction of compactly supported nonstationary dyadic wavelets on the positive half-line. The masks of these wavelets are the Walsh polynomials defined by finite sets of parameters. Application to compression of fractal functions are also discussed.
\end{abstract}

Keywords: Walsh Functions; Nonstationary Dyadic Wavelets; Fractal Functions; Adapted Multiresolution Analysis

\section{Introduction}

As usual, let $\square_{+}=[0,+\infty)$ be the positive half-line, $\square_{+}=\{0,1,2, \cdots\}$ be the set of all nonnegative integers, and let $\square=\{1,2, \cdots\}$ be the set of all positive integers. The first examples of orthogonal wavelets on $\square_{+}$related to the Walsh functions and the corresponding wavelets on the Cantor dyadic group have been constructed in [1]; then, in [2] and [3], a multifractal structure of this wavelets is observed and conditions for wavelets to generate an unconditional basis in $L^{q}$-spaces for all $1<q<\infty$ have been found. These investigations are continued in [4-10] where among other subjects the algorithms to construct orthogonal and biorthogonal wavelets associated with the generalized Walsh functions are studied. In the present paper, using the Walsh-Fourier transform, we construct nonstationary dyadic wavelets on $\square_{+}$(cf. [11-13], [14, Ch.8]).

Let us denote by $[x]$ the integer part of $x$. For every $x \in \square+$, we set

$$
x_{j}=\left[2^{j} x\right](\bmod 2), x_{-j}=\left[2^{1-j} x\right](\bmod 2), j \in \square,
$$

where $x_{j}, x_{-j} \in\{0,1\}$. Then

$$
x=\sum_{j<0} x_{j} 2^{-j-1}+\sum_{j>0} x_{j} 2^{-j} .
$$

The dyadic addition on $\square_{+}$is defined as follows

$$
x \oplus y=\sum_{j<0}\left|x_{j}-y_{j}\right| 2^{-j-1}+\sum_{j<0}\left|x_{j}-y_{j}\right| 2^{-j} .
$$

Further, we introduce the notations

$$
\chi(x, \omega)=(-1)^{\sigma(x, \omega)}, \sigma(x, \omega)=\sum_{j=1}^{\infty} x_{j} \omega_{-j}+x_{-j} \omega_{j},
$$

where $x, \omega \in \square_{+}$. Then the Walsh function $w_{k}$ of order $k$ is $w_{k}(x)=\chi(x, k)$ (see, e.g., [15]).
The Walsh-Fourier transform of every function $f$ that belongs to $L^{1}\left(\square_{+}\right) \cap L^{2}\left(\square_{+}\right)$is defined by

$$
\hat{f}(\omega)=\int_{0}^{\infty} f(x) \chi(x, \omega) \mathrm{d} x, \omega \in \square_{+} .
$$

and extent to the whole space $L^{2}\left(\square_{+}\right)$in a standard way. The intervals

$$
\Delta_{k}^{(n)}=\left[k 2^{-n},(k+1) 2^{-n}\right), \quad k \in \square_{+},
$$

are called the dyadic intervals of range $n$. The dyadic topology on $\square_{+}$is generated by the collection of dyadic intervals. A subset $E$ of $\square_{+}$, which is compact in the dyadic topology will be called $W$-compact.

For any $j \in \square_{+}$we define $\varphi_{j}$ and $\psi_{j}$ by the following algorithm:

Step 1. For each $j \in \square$ choose $n_{j} \in \square$, and $b_{k}^{(j)} \in \square$, $k=0,1 \cdots, 2^{n_{j}}-1$, such that

$$
b_{0}^{(j)}=1,\left|b_{k}^{(j)}\right|^{2}+\left|b_{k+2^{n_{j}-1}}^{(j)}\right|^{2}=1
$$

for all $k=0,1, \cdots, 2^{n_{j}-1}-1$.

Step 2. Define the masks

$$
m_{0}^{(j)}(\omega)=\frac{1}{2} \sum_{k=0}^{2^{n_{j}}-1} c_{k}^{(j)} w_{k}(\omega)
$$

with the coefficients

$$
c_{k}^{(j)}=\frac{1}{2^{n_{j}-1}} \sum_{l=0}^{2^{n_{j}}-1} b_{l}^{(j)} w_{l}\left(2^{-n_{j}} k\right), k=0,1, \cdots, 2^{n_{j}}-1,
$$

so that $m_{0}^{(j)}(\omega)=b_{l}^{(j)}$ for all $\omega \in \Delta_{l}^{(j)}$ (cf. [15, Sect. 9.7]).

Step 3. For each $j \in \square_{+}$put

$$
\hat{\varphi}_{j}(\omega)=2^{-j / 2} \prod_{l=j+1}^{\infty} m_{0}^{(l)}\left(2^{-l} \omega\right),
$$


so that

$$
\varphi_{j}(x)=\frac{1}{\sqrt{2}} \sum_{k=0}^{2^{n_{j}+1}}-1 c_{k}^{(j+1)} \varphi_{j+1}\left(x \oplus 2^{-j-1} k\right) .
$$

Step 4. Define $\psi_{j}$ by the formula

$$
\psi_{j}(x)=\frac{1}{\sqrt{2}} \sum_{k=0}^{2^{n_{j}+1}}(-1)^{k+1} c_{k \oplus 1}^{(j+1)} \varphi_{j+1}\left(x \oplus \frac{k}{2^{j+1}}\right) .
$$

Further, let us define subspaces $\left\{V_{j}\right\}$ and $\left\{W_{j}\right\}$ in $L^{2}\left(\square_{+}\right)$as follows

$$
\begin{aligned}
& V_{j}=\overline{\operatorname{span}\left\{\varphi_{j, k}: k \in \square_{+}\right\}}, \\
& W_{j}=\overline{\operatorname{span}\left\{\psi_{j, k}: k \in \square_{+}\right\}}
\end{aligned}
$$

for all $j \in \square_{+}$.

We say that a polynomial $m$ satisfies the modified Cohen condition if there exists a $W$-compact subset $E$ of $\square+$ such that

$$
\text { int } E \ni 0, \mu(E)=1, E \equiv[0,1)\left(\bmod \square_{+}\right)
$$

and

$$
\inf _{j \in \square} \inf _{\omega \in E}\left|m\left(2^{-j} \omega\right)\right|>0 .
$$

Theorem. Suppose that the masks $m_{0}^{(n)}$ satisfy the modified Cohen condition with a subset $E$ and there exists $j_{0} \in \square$ such that

$$
m_{0}^{(n)}(\omega)=1 \text { for all } \omega \in\left[0,2^{-j_{0}}\right), \quad n \in \square .
$$

Then for any $j \in \square+$ the following properties hold:

a) $\varphi_{j}, \psi_{j} \in L^{2}\left(\square_{+}\right)$and $\operatorname{supp} \varphi_{j} \subset[0,1]$;

b) $\left\{\varphi_{j, k}: k \in \square_{+}\right\}$and $\left\{\psi_{j, k}: k \in \square_{+}\right\}$are orthonormal basis in $V_{j}$ and $W_{j}$, respectively;

c) $V_{j} \subset V_{j+1}, V_{j} \oplus W_{j}=V_{j+1}$.

Moreover, we have

$$
\overline{\bigcup_{j=0}^{\infty} V_{j}}=L^{2}\left(\square_{+}\right) .
$$

Corollary. The system

$$
\left\{\varphi_{0}(\cdot \oplus k): k \in \square_{+}\right\} \cup\left\{\psi_{j, k}: j, k \in \square_{+}\right\}
$$

is an orthonormal basis in $L^{2}\left(\square_{+}\right)$.

We prove this theorem in the next section. Then using the notion of an adapted multiresolution analysis suggested by Sendov [12], we discuss an application of the nonstationary dyadic wavelets to compression of the Weierstrass function and the Swartz function.

\section{Proof of the Theorem}

At first we prove the orthonormality of $\left\{\varphi_{j, k}\right\}_{k \in \square_{+}}$. In view of

$$
\left\langle\varphi_{j, 0}, \varphi_{j, n}\right\rangle=\left\langle\hat{\varphi}_{j, 0}, \hat{\varphi}_{j, n}\right\rangle=\int_{0}^{\infty}\left|\hat{\varphi}_{j}(\omega)\right|^{2} w_{n}\left(2^{-j} \omega\right) \mathrm{d} \omega
$$

let us show that

$$
\int_{0}^{\infty}\left|\varphi_{j}(\omega)\right|^{2} w_{n}\left(2^{-j} \omega\right) \mathrm{d} \omega=\delta_{0, n}, \quad n \in \square_{+} .
$$

Denote by $\mathbf{1}_{E}$ the characteristic function of $E$. For each $j$ we define

$$
\hat{\varphi}_{j}^{(s)}(\omega)=2^{-j / 2} \prod_{l=j+1}^{s} m_{0}^{(l)}\left(2^{-l} \omega\right) \mathbf{1}_{E}\left(2^{-s} \omega\right)
$$

for $s=j+1, j+2, \cdots$ Since $0 \in \operatorname{int} E$ and, for all $j \in \square_{+}$, $m_{0}^{(j)}(\omega)=1$ in some neighbourhood of zero, we obtain from Equation (3)

$$
\lim _{k \rightarrow \infty} \hat{\varphi}_{j}^{(k)}(\omega)=\hat{\varphi}_{j}(\omega) \text { for all } \omega \in \square_{+} \cdot
$$

Let

$$
I_{j}^{(k)}[n]:=\int_{0}^{\infty}\left|\hat{\varphi}_{j}^{(k)}(\omega)\right|^{2} w_{n}\left(2^{-j} \omega\right) \mathrm{d} \omega,
$$

where $k>j, \quad n \in \square_{+}$. Letting $\zeta=2^{-s} \omega$, we have

$$
\begin{aligned}
I_{j}^{(s)}[k] & =2^{s-j} \int_{E} \prod_{l=j+1}^{s}\left|m_{0}^{(l)}\left(2^{s-l} \zeta\right)\right|^{2} w_{k}\left(2^{s-j} \zeta\right) \mathrm{d} \zeta \\
& =2^{s-j} \int_{0}^{1}\left|m_{0}^{(k)}(\zeta)\right|^{2} \prod_{l=j+1}^{s-1}\left|m_{0}^{(l)}\left(2^{s-l} \zeta\right)\right|^{2} w_{k}\left(2^{s-j}\right) \mathrm{d} \zeta \\
& =2^{s-j} \int_{0}^{1 / 2}\left(\left|m_{0}^{(k)}(\zeta)\right|^{2}+\left|m_{0}^{(k)}(\zeta+1 / 2)\right|^{2}\right) \\
& \times \prod_{l=j+1}^{s-1}\left|m_{0}^{(l)}\left(2^{s-l} \zeta\right)\right|^{2} w_{k}\left(2^{s-j} \zeta\right) \mathrm{d} \zeta,
\end{aligned}
$$

that yields $I_{j}^{(s)}[k]=I_{j}^{(s-1)}[k]$. By induction, we obtain

$$
I_{j}^{(s)}[k]=I_{j}^{(s-1)}[k]=\cdots=I_{j}^{(j+1)}[k]=\delta_{0, k} .
$$

According to Equation (8), by Fatou's lemma, we have

$\int_{0}^{\infty}\left|\hat{\varphi}_{j}(\omega)\right|^{2} \mathrm{~d} \omega \leq \lim _{s \rightarrow \infty} \int_{0}^{\infty}\left|\hat{\varphi}_{j}^{(s)}(\omega)\right|^{2} \mathrm{~d} \omega=\lim _{s \rightarrow \infty} I_{j}^{(s)}[0]=1$

Consequently, $\varphi_{j} \in L^{2}\left(\square_{+}\right)$and, in view of Equation (5), $\psi_{j} \in L^{2}\left(\square_{+}\right)$. It is known that if $\hat{f} \in L^{1}\left(\square_{+}\right)$ is constant on dyadic intervals of range $n$, then supp $f \subset\left[0,2^{n}\right]$ (see [16, Sect. 6.2]). Therefore, each function $\hat{\varphi}_{j}$ is constant on $[k, k+1), k \in \square_{+}$, which implies $\operatorname{supp} \varphi_{j} \subset[0,1]$.

In view of Equation (7), there exists $j_{0} \in \square$ such that

$$
m_{0}^{(j)}\left(2^{-j} \omega\right)=1 \text { for all } j>j_{0}, \omega \in E .
$$

Hence, for $\omega \in E$,

$$
\hat{\varphi}_{j}(\omega)=2^{-j / 2} \prod_{l=j+1}^{j_{0}} m_{0}^{(l)}\left(2^{-l} \omega\right) .
$$

It follows from Equation (6) that for some $c_{1}>0$ 


$$
\left|m_{0}^{(j)}\left(2^{-j} \omega\right)\right| \geq c_{1} \quad \text { for } j \in \square, \omega \in E .
$$

Since

$$
c_{1}^{j-j_{0}}\left|\hat{\varphi}_{j}(\omega)\right| \mid \geq 2^{-j / 2} \mathbf{1}_{E}(\omega), \quad \omega \in \square_{+} .
$$

We have

$$
\left|\hat{\varphi}_{j}^{(s)}(\omega)\right| \leq c_{1}^{j-j_{0}} \prod_{l=j+1}^{s}\left|m_{0}^{(l)}\left(2^{-l} \omega\right)\right|\left|\hat{\varphi}_{j}\left(2^{-s} \omega\right)\right| .
$$

or, taking into account Equation (3),

$$
\left|\hat{\varphi}_{j}^{(s)}(\omega)\right|\left|\leq c_{1}^{j-j_{0}}\right| \hat{\varphi}_{j}(\omega) \mid, \quad \omega \in \square_{+}
$$

for $s>j, j \in \square_{+}$.

Applying the dominated convergence theorem we obtain

$$
\begin{aligned}
& \int_{0}^{\infty}\left|\hat{\varphi}_{j}(\omega)\right|^{2} w_{k}\left(2^{-j} \omega\right) \mathrm{d} \omega \\
& =\lim _{s \rightarrow \infty} \int_{0}^{\infty}\left|\hat{\varphi}_{j}^{(s)}(\omega)\right|^{2} w_{k}\left(2^{-j} \omega\right) \mathrm{d} \omega \\
& =\delta_{0, k},
\end{aligned}
$$

which means that $\left\{\varphi_{j, k}\right\}_{k \in \square_{+}}$is an orthonormal system.

Now, let us prove an orthonormality of $\left\{\psi_{j, k}\right\}_{k \in \square_{+}}$.

For any $k \in \square_{+}$denote $\mathrm{d}_{k}^{(j)}=(-1)^{k+1} c_{k \oplus 1}^{(j)}$. Then

$$
{ }_{j, k}(x)=\frac{1}{\sqrt{2}} \sum_{l \in \square_{+}} \mathrm{d}_{l \oplus 2 k}^{(j+1)} \varphi_{j+1, l}(x) .
$$

Since

$$
\psi \sum_{l \in \square_{+}} \mathrm{d}_{l}^{(j)} \mathrm{d}_{l \oplus 2 k}^{(j)}=2 \delta_{0, k}
$$

We have

$$
\begin{aligned}
\left\langle\psi_{j, k}, \psi_{j, k^{\prime}}\right\rangle & =\frac{1}{2} \sum_{l, s \in \square_{+}} \mathrm{d}_{l \oplus 2 k}^{(j+1)} \mathrm{d}_{s \oplus 2 k^{\prime}}^{(j+1)}\left\langle\varphi_{j+1, l}, \varphi_{j+1, s}\right\rangle \\
& =\delta_{k, k^{\prime}} .
\end{aligned}
$$

Then from Equation (10)

$$
V_{j} \subset V_{j+1}, W_{j} \subset V_{j+1} .
$$

Let us define

$$
m_{1}^{(j)}(\omega):=\frac{1}{2} \sum_{k=0}^{2^{n_{j}}-1} \mathrm{~d}_{k}^{(j)} w_{k}(\omega)
$$

Denote $\omega^{\prime}=2^{-j-1} \omega$. Under the unitarity of the matrices

$$
\left(\begin{array}{ll}
m_{0}^{(j)}\left(\omega^{\prime}\right) & m_{0}^{(j)}\left(\omega^{\prime}+1 / 2\right) \\
m_{1}^{(j)}\left(\omega^{\prime}\right) & m_{1}^{(j)}\left(\omega^{\prime}+1 / 2\right)
\end{array}\right)
$$

We can write

$$
\begin{aligned}
& \hat{\varphi}_{j+1}(\omega)=\hat{\varphi}_{j+1}(\omega) \\
& \times\left\{\left[\left|m_{0}^{(j+1)}\left(\omega^{\prime}\right)\right|^{2}+\left|m_{1}^{(j+1)}\left(\omega^{\prime}\right)\right|^{2}\right]\right. \\
& +\left[m_{0}^{(j+1)}\left(\omega^{\prime}\right) \overline{m_{0}^{(j+1)}\left(\omega^{\prime}+1 / 2\right)}\right. \\
& \left.\left.+m_{1}^{(j+1)}\left(\omega^{\prime}\right) \overline{m_{1}^{(j+1)}\left(\omega^{\prime}+1 / 2\right)}\right]\right\} \\
& =\left[\overline{m_{0}^{(j+1)}\left(\omega^{\prime}\right)}+\overline{m_{0}^{(j+1)}\left(\omega^{\prime}+1 / 2\right)}\right] \\
& \times m_{0}^{(j+1)}\left(\omega^{\prime}\right) \hat{\varphi}_{j+1}(\omega) \\
& +\left[\overline{m_{1}^{(j+1)}\left(\omega^{\prime}\right)} \overline{m_{1}^{(j+1)}\left(\omega^{\prime}+1 / 2\right)}\right] \\
& \times m_{1}^{(j+1)}\left(\omega^{\prime}\right) \hat{\varphi}_{j+1}(\omega) \\
& =\sqrt{2} \sum_{l \in \square} \bar{c}_{2 l}^{(j+1)} w_{2 l}\left(2^{-j-1} \omega\right) \hat{\varphi}_{j}(\omega) \\
& +\sqrt{2} \sum_{l \in \square} \bar{d}_{2 l}^{(j+1)} w_{2 l}\left(2^{-j-1} \omega\right) \hat{\psi}_{j}(\omega) .
\end{aligned}
$$

Using the inverse Fourier-Walsh transform, we have

$$
\varphi_{j+1}(x)=\sqrt{2} \sum_{l \in \square_{+}}\left(\bar{c}_{2 l}^{(j+1)} \varphi_{j, l}(x)+\overline{\mathrm{d}}_{2 l}^{(j+1)} \psi_{j, l}(x)\right)
$$

or,

$$
\varphi_{j+1, k}(x)=\sqrt{2} \sum_{l \in \square_{+}}\left(\bar{c}_{k \oplus 2 l}^{(j+1)} \varphi_{j, l}(x)+\overline{\mathrm{d}}_{k \oplus 2 l}^{(j+1)} \psi_{j, l}(x)\right) .
$$

With Equation (11) it yields $V_{j} \oplus W_{j}=V_{j+1}$

To conclude the proof it remains to show that

$$
\overline{\bigcup_{j=0}^{\infty} V_{j}}=L_{2}\left(\square_{+}\right) \text {. }
$$

Note, that by Equation (7) for any $\omega \in \square+$ there exist $j \in \square+$ such that $\hat{\varphi}_{j}(\omega)=2^{-j / 2}$ and, consequently,

$$
\bigcup_{j=0}^{\infty} \operatorname{supp} \hat{\varphi}_{j}=\square_{+} \text {. }
$$

For any $x \in \square_{+}$the subspace $\overline{\bigcup_{j=0}^{\infty} V_{j}}$ is invariant with respect to the shift $f(\cdot) \mapsto f(\cdot \oplus x)$. Actually, an arbitrary $x \in \square+$ can be approximated by fractions $2^{-j} l$, with arbitrary large $j$. Besides, each $V_{j}$ is invariant with respect to the shifts $2^{-j} l$. By Equation (4) it is clear that $V_{j} \subset V_{j+1}$.

Let $f \in \bigcup_{j=0}^{\infty} V_{j}$. There exist $j_{1}$ such that $f \in V_{j_{1}}$ and hence $f\left(\cdot \oplus 2^{-j} l\right) \in V_{j} \quad$ for all $j \geq j_{1}$. The continuity of $\|f(\cdot \oplus x)\|$ implies that $f(\cdot \oplus x) \in \overline{\bigcup_{j=0}^{\infty} V_{j}}$. If $g \in \overline{\bigcup_{j=0}^{\infty} V_{j}}$, then approximating $g$ with $f$ from $\bigcup_{j=0}^{\infty} V_{j}$ and using the invariance of a norm with respect to the shift, we obtain $g(\cdot \oplus x) \in \overline{\bigcup_{j=0}^{\infty} V_{j}}$. 
Denote by $\left(\overline{\bigcup_{j=0}^{\infty} V_{j}}\right)^{\wedge}$ the set of all $\hat{f}$ such that $f \in \bigcup_{j=0}^{\infty} V_{j}$. By the Weiner's theorem we can write $\left(\overline{\bigcup_{j=0}^{\infty} V_{j}}\right)^{\wedge}=L_{2}(\Omega)$, for some measurable $\Omega \subset \square_{+}$. It is clearly that $\bigcup_{j=0}^{\infty} \operatorname{supp} \hat{\varphi}_{j} \subset \Omega$ and, in view of Equation (13), we have $\Omega=\square_{+}$. Hence, the Equation (12) holds. The theorem is proved.

\section{Numerical Experiments}

For any $N \in \square$, let $\Delta_{j}(N):=\left[0,(2 N-1) 2^{-j}\right], \quad j \in \square_{+}$. According to [12] an adapted multiresolution analysis (AMRA) of rank $N$ in $L^{2}(\square)$ is a collection of closed subspaces $V_{j} \subset L^{2}(\square), \quad j \in \square_{+}$, which satisfies the following conditions:

1) $V_{j} \subset V_{j+1}$ for all $j \in \square_{+}$;

2) $\overline{\bigcup_{j=0}^{\infty} V_{j}}=L^{2}(\square)$;

3) For every $j \in \square_{+}$there is a function $\varphi_{j}$ in $L^{2}(\square)$ with a finite support $\Delta_{j}(N)$ such that $\left\{\varphi_{j}\left(\cdot-k 2^{-j}\right): k \in \square\right\}$ is an orthonormal basis of $V_{j}$;

4) For every $j \in \square_{+}$there exists a filter

$$
\mathbf{c}(j)=\left\{c_{k}(j)\right\}_{k=0}^{2 N-1}
$$

such that

$$
\varphi_{j-1}(x)=\sum_{k=0}^{2 N-1} c_{k}(j) \varphi_{j}\left(x-k 2^{-j}\right), \quad j \in \square .
$$

The sequence $\left\{\varphi_{j}\right\}$ from condition (4) is called a scaling sequence for given an AMRA. The corresponding a wavelet sequence $\left\{\psi_{j}\right\}$ can be defined by

$$
\psi_{j-1}(x)=\sum_{k=0}^{2 N-1}(-1)^{k} c_{2 N-k-1}(j) \varphi_{j}\left(x-k 2^{-j}\right) .
$$

Denote by $W_{j}$ the orthogonal complement of $V_{j-1}$ in $V_{j}$. It is known that, under some conditions, the system $\left\{\psi_{j}\left(\cdot-k 2^{-j}\right): k \in \square\right\}$ is an orthonormal basis of $W_{j}$ (for more details, see, e.g., [14, Sect. 8.1]). Moreover, if $f_{A}$ denotes the projection of a function $f \in L^{2}(\square)$ on the subset $A \subset L^{2}(\square)$, then

$$
\|f\|^{2}=\left\|f_{V_{0}}\right\|^{2}+\sum_{j=0}^{\infty}\left\|f_{W_{j}}\right\|^{2}
$$

and

$$
\left\|f_{V_{j}}\right\|^{2}=\left\|f_{V_{j-1}}\right\|^{2}+\left\|f_{W_{j-1}}\right\|^{2} .
$$

Let us denote

$$
h_{k}(j)=c_{k}(j) / \sqrt{2}
$$

and

$$
g_{k}(j)=(-1)^{k} h_{1-k}(j) .
$$

For a given array

$$
\mathbf{A}(j)=\left\{a_{j, 0}, a_{j, 1} \cdots, a_{j, 2^{j}-1}\right\},
$$

the direct non-stationary discrete wavelet transform

$$
a_{j-1, k}=\sum_{l \in \llbracket} h_{l-2 k}(j) a_{j, l}, d_{j-1, k}=\sum_{l \in \llbracket} g_{l-2 k}(j) a_{j, l},
$$

maps it into

$$
\mathbf{A}(j-1)=\left\{a_{j-1,0}, a_{j-1,1} \cdots, a_{j-1,2^{j-1}-1}\right\}
$$

and

$$
\mathbf{D}(j-1)=\left\{a_{j-1,0}, a_{j-1,1} \cdots, a_{j-1,2^{j-1}-1}\right\} .
$$

The inverse transform is defined as follows

$$
a_{j, l}=\sum_{k \in \square} h_{l-2 k}(j) a_{j-1, l}+g_{l-2 k}(j) d_{j-1, l}
$$

and reconstructs $\mathbf{A}(j)$ by $\mathbf{A}(j-1)$ and $\mathbf{D}(j-1)$. According to [12] in order to choose the filter $\mathbf{c}(j)$ to maximize $\left\|f_{V_{j-1}}\right\|^{2}$ in Equation (16), we must solve the following problem.

Problem 1. Let $U_{N}^{(1)}$ be the subset of the $2 N$-dimensional Euclidean space $\square^{2 N}$, which consists of the points $u=\left(u_{0}, u_{1}, \cdots, u_{2 N-1}\right)$ satisfying the conditions

$$
\sum_{k=0}^{2 N-1} u_{k}^{2}=1, \sum_{k=0}^{2 N-l-1} u_{k} u_{2 l+k}=0
$$

for $l=0,1 \cdots, N-1$. Find a point $u^{*}$ for which

$$
\sum_{m, k=0}^{2 N-1} u_{m}^{*} u_{k}^{*} F_{m, k}=\sup _{u \in U_{N}^{(1)}}\left\{\sum_{m, k=0}^{2 N-1} u_{m} u_{k} F_{m, k}\right\},
$$

where $\left\|F_{m, k}\right\|$ is a $2 N \times 2 N$ symmetric matrix.

Problem 1 has a solution since $U_{N}$ is a compact. But, as noted in [12], the numerical solution of this problem is not trivial even for $N=2$.

Concerning the standard Haar and Daubechies (with 4 coefficients) discrete transforms see, e.g., [17]; we will denote them as SWTH and SWTD, respectively. We write NSWTH for the simplest case of a multiresolution analysis of rank 1 which is considered in [12, Sect. 3] (see also [13]). The nonstationary Daubechies discrete wavelet transform which corresponds an AMRA of rank $N$ are defined in [12] and we will use the symbol NSWTDN to denote this transform (see NSWTD1 and NSWTD2 in the tables below).

Method A associated with one of the mentioned above discrete wavelet transforms (cf. [17, Chap.7]) consists of the following steps: 
Step 1. Apply the discrete wavelet transform $j$ times to an input array $\mathbf{A}(j)$ and get the sequence

$$
\mathbf{A}(0), \mathbf{D}(0), \mathbf{D}(1), \cdots, \mathbf{D}(j-1) \text {. }
$$

Step 2. Allocate a certain percentage of the wavelet coefficients with lagest absolute value (we choose 10\%) and nullify the remaining coefficients.

Step 3. Apply the inverse wavelet transform to the modified arrays of the wavelet coefficients.

Step 4. Calculate $\|\mathbf{A}(j)-\tilde{\mathbf{A}}(j)\|_{2}$, where $\mathbf{A}(j)$ is a reconstructed array.

In Method B the second step is replaced on the uniform quantization and the forth step is replaced on the calculation of the entropy of a vector, obtained in the third step.

We recall that $\mathbf{y}=\left\{y_{1}, \cdots, y_{m}\right\}$ is a vector uniform quantization for given vector $\mathbf{x}=\left(x_{1}, \cdots, x_{m}\right)$, if

$$
y_{j}=\left\{\begin{array}{l}
0,\left|x_{j}\right|<\Delta, \\
\Delta\left[\frac{x_{j}}{\Delta}\right]+\operatorname{sign}\left(x_{j}\right) \frac{\Delta}{2},\left|x_{j}\right| \geq \Delta,
\end{array}\right.
$$

where $\Delta$ is the length of the quantization interval.

The value $\Delta$ will be calculated by

$$
\Delta=\left(\max _{1 \leq j \leq m} x_{j}-\min _{1 \leq j \leq m} x_{j}\right) / 50 .
$$

The Shannon entropy of $\mathbf{x}$ is defined by the formula

$$
H(\mathbf{x})=-\sum_{j=1}^{m} p_{j} \log _{2}\left(p_{j}\right),
$$

where $p_{j}$ is frequency of the value $x_{j}$.

Let us consider a similar approach associated with the following problem:

Problem 2. Let $N=2^{n-1}$. Denote by $U_{N}^{(2)}$ the set of all points $u=\left(u_{0}, u_{1}, \cdots, u_{2 N-1}\right) \in \square^{2 N}$ such that

$$
\left(u_{l}\right)^{2}+\left(u_{l+N}\right)^{2}=1, l=0,1, \cdots, N-1 .
$$

For every $u \in U_{N}^{(2)}$ we define

$$
c_{k}(u)=\frac{1}{N} \sum_{j=0}^{2 N-1} u_{j} w_{j}(k /(2 N))
$$

for $k=0,1, \cdots, 2 N-1$. Find a point $u^{*}$ for which

$$
\begin{aligned}
& \sum_{m, k=0}^{2 N-1} c_{m}\left(u^{*}\right) c_{k}\left(u^{*}\right) F_{m, k} \\
& =\sup _{u \in U_{N}^{(2)}}\left\{\sum_{m, k=0}^{2 N-1} c_{m}(u) c_{k}(u) F_{m, k}\right\},
\end{aligned}
$$

where $\left\|F_{m, k}\right\|$ is a $2 N \times 2 N$ symmetric matrix.

Given an array $\mathbf{A}(j)=\left\{a_{j, 0}, a_{j, 1} \cdots, a_{j, 2^{j}-1}\right\}$, we define the matrix $\left\|F_{m, k}\right\|$ in Problem 1 and Problem 2 by

$$
F_{m, k}=\sum_{s \in \square} a_{j, 2 s+m} a_{j, 2 s+m}
$$

and

$$
F_{m, k}=\sum_{s \in \square_{+}} a_{j, 2 s \oplus m} a_{j, 2 s \oplus m},
$$

respectively. Here $a_{j, s}=0$ for $s \notin\left\{0,1, \cdots, 2^{j}-1\right\}$. Suppose that $u^{*}$ is a solution of Equation (19). Then the direct and inverse nonstationary discrete dyadic wavelet transforms are defined by

$$
\begin{aligned}
& a_{j-1, k}=\sum_{l \in \square_{+}} h_{l \oplus 2 k}^{(j)} a_{j, l}, \quad d_{j-1, k}=\sum_{l \in \square+} g_{l \oplus 2 k}^{(j)} a_{j, l}, \\
& a_{j, l}=\sum_{k \in \square_{+}} h_{l \oplus 2 k}^{(j)} a_{j-1, l}+g_{l \oplus 2 k}^{(j)} d_{j-1, l},
\end{aligned}
$$

where $h_{k}^{(j)}=c_{k}\left(u^{*}\right) / \sqrt{2}$ and $g_{k}^{(j)}=(-1)^{k} h_{1 \oplus k}^{(j)}$. We

Table 1. Values of the square error corresponding to Method A.

\begin{tabular}{llllllll}
\hline & SWTH & NSWTH & NSWTL1 & SWTD & NSWTD1 & NSWTD2 & NSWTL2 \\
\hline $\mathcal{S}$ & 0.166547 & 0.123983 & 0.123980 & 0.248311 & 0.167071 & 0.128120 & 0.122886 \\
$\mathcal{W}_{0.9,3}$ & 15.823238 & 14.802541 & 14.802635 & 14.290849 & 14.807025 & 14.275246 & 14.022471 \\
$\mathcal{W}_{0.9,5}$ & 16.813738 & 15.932313 & 15.932307 & 15.378600 & 15.171461 & 14.782221 & 15.130797 \\
$\mathcal{W}_{0.9,7}$ & 15.887306 & 13.631379 & 13.631383 & 15.595433 & 16.649683 & 12.724437 & 12.674001 \\
\hline
\end{tabular}

Table 2. Values of the entropy obtained by Method B.

\begin{tabular}{llllllll}
\hline & SWTH & NSWTH & NSWTL1 & SWTD & NSWTD1 & NSWTD2 & NSWTL2 \\
\hline $\mathcal{S}$ & 0.320865 & 0.327626 & 0.310639 & 0.863949 & 0.299818 & 0.304681 & 0.241210 \\
$\mathcal{W}_{0.9,3}$ & 4.486757 & 3.810555 & 3.772764 & 4.152313 & 3.822598 & 3.525294 & 3.466450 \\
$\mathcal{W}_{0.9,5}$ & 4.688737 & 3.874187 & 3.848227 & 4.224801 & 4.106692 & 3.766994 & 3.700762 \\
$\mathcal{W}_{0.9,7}$ & 4.392570 & 3.371864 & 3.344916 & 4.001358 & 4.435942 & 3.232151 & 3.197167 \\
\hline
\end{tabular}


denote these discrete transforms as NSWTL1 if $N=1$ and as NSWTL2 if $N=2$.

Let us recall that the Weierstrass function is defined as

$$
\mathcal{W}_{\alpha, \beta}(x)=\sum_{n=1}^{\infty} \alpha^{n} \cos \left(\beta^{n} \pi x\right), 0<\alpha<1, \beta \geq \frac{1}{\alpha},
$$

and the Swartz function is defined as

$$
\mathcal{S}(x)=\sum_{n=1}^{\infty} \frac{h\left(2^{n} x\right)}{4^{n}}
$$

where $h(x)=[x]-\sqrt{x-[x]}$. We will consider arrays A(8) with elements $a_{8, k}=\mathcal{W}_{\alpha, \beta}(k / 128)$ or $a_{8, k}=$ $\mathcal{S}(k / 256), \quad k=0, \cdots, 255$. Then we use the Matlab function fminsearch to solve the optimization problems in Equations (18) and (19). The results of these numerical experiments are presented in Tables 1 and 2 . We see that in several cases the introduced nonstationary dyadic wavelets have an advantage over the classical Haar and Daubechies wavelets.

\section{REFERENCES}

[1] W. C. Lang, "Orthogonal Wavelets on the Cantor Dyadic Group," SIAM Journal on Mathematical Analysis, Vol. 27, No. 1, 1996, pp. 305-312. doi:10.1137/S0036141093248049

[2] W. C. Lang, "Wavelet Analysis on the Cantor Dyadic Group," Houston Journal of Mathematics, Vol. 24, No. 3, 1998, pp. 533-544.

[3] W. C. Lang, "Fractal Multiwavelets Related to the Cantor Dyadic Group," International Journal of Mathematics and Mathematical Sciences, Vol. 21, No. 2, 1998, pp. 307-317. doi:10.1155/S0161171298000428

[4] Y. A. Farkov, "Orthogonal Wavelets with Compact Support on Locally Compact Abelian Groups," Izvestiya: Mathematics, Vol. 69, No. 3, 2005, pp. 623-650. doi:10.1070/IM2005v069n03ABEH000540

[5] Y. A. Farkov, "Wavelets and Frames in Walsh Analysis," In: M. del Valle, Ed., Wavelets: Classification, Theory and Applications, Chapter 11, Nova Science Publishers, New York, 2012, pp. 267-304.

[6] Y. A. Farkov and E. A. Rodionov, "Estimates of the
Smoothness of Dyadic Orthogonal Wavelets of Daubechies Type," Mathematical Notes, Vol. 82, No. 6, 2007, pp. 407-421. doi:10.1134/S0001434609090144

[7] Y. A. Farkov, A. Yu. Maksimov and S. A. Stroganov, "On Biorthogonal Wavelets Related to the Walsh Functions," International Journal of Wavelets, Multiresolution and Information Processing, Vol. 9, No. 3, 2011, pp. 485 499. doi:10.1142/S0219691311004195

[8] Y. A. Farkov and E. A. Rodionov, "Algorithms for Wavelet Construction on Vilenkin Groups," P-Adic Numbers, Ultrametric Analysis, and Applications, Vol. 3, No. 3, 2011, pp. 181-195. doi:10.1134/S2070046611030022

[9] Y. A. Farkov, "Periodic Wavelets on the p-Adic Vilenkin Group," P-Adic Numbers, Ultrametric Analysis, and Applications, Vol. 3, No. 4, 2011, pp. 281-287. doi:10.1134/S2070046611040030

[10] Y. A. Farkov and M. E. Borisov, "Periodic Dyadic Wavelets and Coding of Fractal Functions," Russian Mathematics (Izvestiya VUZ. Matematika), No. 9, 2012, pp. 5465.

[11] I. Y. Novikov, "On the Construction of Nonstationary Orthonormal Infinitely Differentiable Compactly Supported Wavelets," Proceedings of the 12th International Association for Pattern Recognition, Jerusalem, 9-13 October 1994, pp. 214-215. doi:10.1109/ICPR.1994.577164

[12] B. Sendov, "Adapted Multiresolution Analysis," Proceedings of Alexits Memorial Conference Functions, Series, Operators, Budapest, 9-14 August 1999, pp. 23-38.

[13] B. Sendov, "Adaptive Multiresolution Analysis on the Dyadic Topological Group," Journal of Approximation Theory, Vol. 96, No. 2, 1998, pp. 21-45. doi:10.1006/jath.1998.3234

[14] I. Y. Novikov, V. Y. Protasov and M. A. Skopina, "Wavelet Theory," American Mathematical Society, Providence, 2011.

[15] F. Schipp, W. R. Wade and P. Simon, "Walsh Series: An Introduction to Dyadic Harmonic Analysis," Adam Hilger, Bristol, 1990.

[16] B. I. Golubov, A. V. Efimov and V. A. Skvortsov, "Walsh Series and Transforms," Kluwer, Dordrecht, 1991.

[17] S. Welstead, "Fractal and Wavelet Image Compression Techniques," SPIE Optical Engineering Press, Bellingham, 2002. 